The method of matrix elements' calculations for the Dirac equation in the Coulomb field

This content has been downloaded from IOPscience. Please scroll down to see the full text.

2011 J. Phys. B: At. Mol. Opt. Phys. 44045002

(http://iopscience.iop.org/0953-4075/44/4/045002)

View the table of contents for this issue, or go to the journal homepage for more

Download details:

IP Address: 195.19.236.163

This content was downloaded on 02/06/2015 at 16:08

Please note that terms and conditions apply. 


\title{
The method of matrix elements' calculations for the Dirac equation in the Coulomb field
}

\author{
A M Puchkov \\ Theoretical Department, Institute of Physics, St Petersburg State University, 198904 Petergof, \\ St Petersburg, Russia \\ E-mail: putchkov@mail.ru and putchkov@yahoo.com \\ Received 25 October 2010, in final form 23 November 2010 \\ Published 28 January 2011 \\ Online at stacks.iop.org/JPhysB/44/045002

\begin{abstract}
The method of matrix elements' calculations for the Dirac equation in the Coulomb field based on the virial relations is suggested. A matrix representation of virial relations for the Dirac equation in the Coulomb field is given. An explicit form of matrices in the cases of direct and inverse recursion is found. Possible applications of the suggested method are discussed and the probability of forbidden M1 transitions is calculated as an example.
\end{abstract}

\section{Introduction}

We consider a set of states of a relativistic particle in a central field. It was shown in [1-7] that some of the matrix elements of these states are connected by recurrent relations. These relations were named virial because the virial theorem was used to deduce them. The idea to use some of them in case of Coulomb field for summation of perturbation series was introduced in a paper [5] (see also a review [6]) for the first time. Computations have shown that this method is more effective than the use of the reduced CoulombGreen functions. Later the virial relations were repeatedly and successfully used for the analytical and high-precision numerical calculations of the hyperfine splitting and the boundelectron $g$-factor in H-like ions (see, for example, [8-10] and references therein).

However, although these relations have been well known for a rather long time, a number of important and fundamental issues are still unsolved. In particular a so-called problem of negative powers and the problem of the condition of applicability should be mentioned.

The aim of this paper is to elaborate and simplify the mathematical apparatus of virial relations for Dirac equations in the Coulomb field that will provide solutions for the problems mentioned above. Besides, this work allows us to obtain simple formulae for the calculation of matrix elements for suitable powers $s$.

In recent papers $[11,12]$ the diagonal matrix elements $\left\langle O r^{s}\right\rangle$, where $O=\{1, \beta, \imath \alpha \boldsymbol{n} \beta\}$ are the standard Dirac matrix operators and angular brackets denote the quantum mechanical average for the relativistic Coulomb problem, have been evaluated as sums of three specialized hypergeometric functions ${ }_{3} F_{2}(1)$. In these works it was also shown that the set of matrix elements $\left\langle O r^{s}\right\rangle$ is connected with the set $\left\langle O r^{s-1}\right\rangle$ for all suitable powers $s$ by using $3 \times 3$ matrices. In this paper we consider the general case of non-diagonal matrix elements and show that virial relations among them can be written down in terms of $4 \times 4$ matrices.

The paper consists of three parts. The first one is of introductory character and contains the derivation and comparison of the known results. In the second section, we set the mathematical structure of virial relations and explicit expressions of matrix elements for direct and inverse recursion. In the third section, by calculating the probability of forbidden M1 transitions in hydrogen atom and light $\mathrm{H}$-like ions, it is shown how to apply the mathematical apparatus developed here to particular problems. In this paper relativistic units ( $\hbar=c=1$ ) are used, and the terminology and notation are taken from [5] and [6].

\section{Virial relations for the Dirac equation in a central field}

For the case of a central field $V(r)$, the stationary Dirac equation has the form

$$
(-\imath \boldsymbol{\alpha} \cdot \nabla+\beta m+V(r)) \psi(\mathbf{r})=E \psi(\mathbf{r}),
$$


The wavefunction is conveniently represented by

$$
\psi(\mathbf{r})=\left(\begin{array}{c}
g_{n \kappa}(r) \Omega_{\kappa m}(\mathbf{n}) \\
l f(r)_{n \kappa} \Omega_{-\kappa m}(\mathbf{n})
\end{array}\right),
$$

where $\Omega_{ \pm \kappa m}(\mathbf{n})$ is the spinor spherical harmonics, $\mathbf{n}=$ $\mathbf{n}(\vartheta, \varphi)=\mathbf{r} / r$, and $\kappa=(-1)^{j+l+1 / 2}(j+1 / 2)$ is the quantum number determined by the angular momentum and the parity of state. Substitute (2) into (1) and define $G_{n \kappa}(r)=$ $r g_{n \kappa}(r), F_{n \kappa}(r)=r f_{n \kappa}(r)$. After separation of variables, one can obtain the following system for radial functions:

$$
\begin{aligned}
& \frac{\mathrm{d} G}{\mathrm{~d} r}+\frac{\kappa}{r} G-(E+m-V) F=0, \\
& \frac{\mathrm{d} F}{\mathrm{~d} r}-\frac{\kappa}{r} F+(E-m-V) G=0,
\end{aligned}
$$

Rewrite (3)-(4) in a compact form. Then the radial functions $G_{n \kappa}(r)$ and $F_{n \kappa}(r)$ should be collected into the column

$$
\phi_{n \kappa}(r)=\left(\begin{array}{c}
G_{n \kappa}(r) \\
F_{n \kappa}(r)
\end{array}\right),
$$

and according to [13] the following operator can be introduced:

$$
H_{\kappa}=-\imath \sigma_{y} \frac{\mathrm{d}}{\mathrm{d} r}+\sigma_{x} \frac{\kappa}{r}+\sigma_{z} m+V,
$$

where $\sigma_{x}, \sigma_{y}$ and $\sigma_{z}$ are the Pauli matrices. Hence,

$$
H_{\kappa} \phi_{n \kappa}(r)=E_{n \kappa} \phi_{n \kappa}(r) \text {. }
$$

Obviously the functions $\phi_{n \kappa}(r)$ satisfy the following boundary conditions:

$$
\left.\phi_{n \kappa}(r)\right|_{r=0}=0, \quad\left|\phi_{n \kappa}(r)\right|_{r \rightarrow \infty}^{\longrightarrow} 0 .
$$

Introduce the symbols for matrix elements:

$$
\begin{aligned}
& X_{0}(s)=\left\langle n^{\prime} \kappa^{\prime}\left|r^{s}\right| n \kappa\right\rangle=\int_{0}^{\infty}\left(F_{n^{\prime} k^{\prime}} F_{n k}+G_{n^{\prime} k^{\prime}} G_{n k}\right) r^{s} \mathrm{~d} r, \\
& X_{1}(s)=\left\langle n^{\prime} \kappa^{\prime}\left|\sigma_{x} r^{s}\right| n \kappa\right\rangle=\int_{0}^{\infty}\left(F_{n^{\prime} k^{\prime}} G_{n k}+G_{n^{\prime} k^{\prime}} F_{n k}\right) r^{s} \mathrm{~d} r,
\end{aligned}
$$

$X_{2}(s)=\left\langle n^{\prime} \kappa^{\prime}\left|\imath \sigma_{y} r^{s}\right| n \kappa\right\rangle=\int_{0}^{\infty}\left(G_{n^{\prime} k^{\prime}} F_{n k}-F_{n^{\prime} k^{\prime}} G_{n k}\right) r^{s} \mathrm{~d} r$

$X_{3}(s)=\left\langle n^{\prime} \kappa^{\prime}\left|\sigma_{z} r^{s}\right| n \kappa\right\rangle=\int_{0}^{\infty}\left(G_{n^{\prime} k^{\prime}} G_{n k}-F_{n^{\prime} k^{\prime}} F_{n k}\right) r^{s} \mathrm{~d} r$.

It was shown in [5] that matrix elements commutators and anticommutators of the operator $H_{\kappa}$ with $r^{s}, \sigma_{x} r^{s}, \iota \sigma_{y} r^{s}$ and $\sigma_{z} r^{s}$ could be designed via the following system of equations:

$$
\begin{aligned}
& \left(E_{n^{\prime} \kappa^{\prime}}-E_{n \kappa}\right)\left\langle n \kappa^{\prime}\left|r^{s}\right| n \kappa\right\rangle \\
& \quad=-s\left\langle n^{\prime} \kappa^{\prime}\left|\iota \sigma_{y} r^{s-1}\right| n \kappa\right\rangle+\left(\kappa^{\prime}-\kappa\right)\left\langle n^{\prime} \kappa^{\prime}\left|\sigma_{x} r^{s-1}\right| n \kappa\right\rangle,
\end{aligned}
$$

$$
\begin{aligned}
\left(E_{n^{\prime} \kappa^{\prime}}\right. & \left.-E_{n \kappa}\right)\left\langle n^{\prime} \kappa^{\prime}\left|l \sigma_{y} r^{s}\right| n \kappa\right\rangle \\
= & s\left\langle n^{\prime} \kappa^{\prime}\left|r^{s-1}\right| n \kappa\right\rangle-\left(\kappa^{\prime}+\kappa\right)\left\langle n^{\prime} \kappa^{\prime}\left|\sigma_{z} r^{s-1}\right| n \kappa\right\rangle \\
& +2 m\left\langle n^{\prime} \kappa^{\prime}\left|\sigma_{x} r^{s}\right| n \kappa\right\rangle,
\end{aligned}
$$

$$
\begin{aligned}
\left(E_{n^{\prime} \kappa^{\prime}}\right. & \left.+E_{n \kappa}\right)\left\langle n^{\prime} \kappa^{\prime}\left|\sigma_{z} r^{s}\right| n \kappa\right\rangle \\
= & s\left\langle n^{\prime} \kappa^{\prime}\left|\sigma_{x} r^{s-1}\right| n \kappa\right\rangle-\left(\kappa^{\prime}-\kappa\right)\left\langle n^{\prime} \kappa^{\prime}\left|l \sigma_{y} r^{s-1}\right| n \kappa\right\rangle \\
& +2 m\left\langle n^{\prime} \kappa^{\prime}\left|r^{s}\right| n \kappa\right\rangle+2\left\langle n^{\prime} \kappa^{\prime}\left|\sigma_{z} V r^{s}\right| n \kappa\right\rangle,
\end{aligned}
$$

$$
\begin{aligned}
\left(E_{n^{\prime} \kappa^{\prime}}\right. & \left.+E_{n \kappa}\right)\left\langle n^{\prime} \kappa^{\prime}\left|\sigma_{x} r^{s}\right| n \kappa\right\rangle \\
= & -s\left\langle n^{\prime} \kappa^{\prime}\left|\sigma_{z} r^{s-1}\right| n \kappa\right\rangle+\left(\kappa^{\prime}+\kappa\right)\left\langle n^{\prime} \kappa^{\prime}\left|r^{s-1}\right| n \kappa\right\rangle \\
& +2\left\langle n^{\prime} \kappa^{\prime}\left|\sigma_{x} V r^{s}\right| n \kappa\right\rangle .
\end{aligned}
$$

Equations (10)-(13) are named the virial relations. It should be mentioned that there exists a simpler way to obtain them. The equations for matrix elements (6)-(9) should be integrated by parts; then expressions (3)-(4) should be used with boundary conditions (5). Therefore calling equations (10)-(13) virial is no more than a tradition. Attention should also be drawn to the fact that equations (10) and (11) do not contain the potential $V(r)$.

\section{Mathematical structure of virial relations in the Coulomb field}

Let us consider a charged particle moving in the Coulomb field $V=-\alpha Z / r$. In this case equations (10)-(13) are twoterm recurrence relations. We shall explicitly present their structure.

Let us suppose that $s$ is a positive integer number. We put the matrix element containing $r^{s}$ on the left-hand side of every equation, and on the right-hand side we put a linear combination of matrix elements containing $r^{s-1}$. Equations (10) and (13) can be simply transformed into

$$
\begin{aligned}
X_{0}(s)= & \frac{\left(\kappa^{\prime}-\kappa\right)}{\left(E_{n^{\prime} \kappa^{\prime}}-E_{n \kappa}\right)} X_{1}(s-1) \\
& -\frac{s}{\left(E_{n^{\prime} \kappa^{\prime}}-E_{n \kappa}\right)} X_{2}(s-1) \\
X_{1}(s)= & \frac{\left(\kappa^{\prime}+\kappa\right)}{\left(E_{n^{\prime} \kappa^{\prime}}+E_{n \kappa}\right)} X_{0}(s-1)-\frac{2(\alpha Z)}{\left(E_{n^{\prime} \kappa^{\prime}}+E_{n \kappa}\right)} X_{1}(s-1) \\
& -\frac{s}{\left(E_{n^{\prime} \kappa^{\prime}}+E_{n \kappa}\right)} X_{3}(s-1) .
\end{aligned}
$$

Let us substitute the expression for the matrix element $X_{1}(s)$ from (15) into equation (11):

$$
\begin{aligned}
X_{2}(s)= & {\left[\frac{s}{\left(E_{n^{\prime} \kappa^{\prime}}-E_{n \kappa}\right)}+\frac{2 m\left(\kappa^{\prime}+\kappa\right)}{\left(E_{n^{\prime} \kappa^{\prime}}^{2}-E_{n \kappa}^{2}\right)}\right] X_{0}(s-1) } \\
& -\frac{4 m(\alpha Z)}{\left(E_{n^{\prime} \kappa^{\prime}}^{2}-E_{n \kappa}^{2}\right)} X_{1}(s-1) \\
& -\left[\frac{\left(\kappa^{\prime}+\kappa\right)}{\left(E_{n^{\prime} \kappa^{\prime}}-E_{n \kappa}\right)}+\frac{2 m s}{\left(E_{n^{\prime} \kappa^{\prime}}^{2}-E_{n \kappa}^{2}\right)}\right] X_{3}(s-1) .
\end{aligned}
$$


Then we substitute the expression for the matrix element $X_{0}(s)$ from (14) into equation (12) and obtain

$$
\begin{aligned}
X_{3}(s)= & {\left[\frac{s}{\left(E_{n^{\prime} \kappa^{\prime}}+E_{n \kappa}\right)}+\frac{2 m\left(\kappa^{\prime}-\kappa\right)}{\left(E_{n^{\prime} \kappa^{\prime}}^{2}-E_{n \kappa}^{2}\right)}\right] X_{1}(s-1) } \\
& -\left[\frac{\left(\kappa^{\prime}-\kappa\right)}{\left(E_{n^{\prime} \kappa^{\prime}}+E_{n \kappa}\right)}+\frac{2 m s}{\left(E_{n^{\prime} \kappa^{\prime}}^{2}-E_{n \kappa}^{2}\right)}\right] X_{2}(s-1) \\
& -\frac{2(\alpha Z)}{\left(E_{n^{\prime} \kappa^{\prime}}+E_{n \kappa}\right)} X_{3}(s-1) .
\end{aligned}
$$

Obviously equations (14)-(17) can now be represented in matrix form:

$$
\mathbf{X}(s)=A(s) \mathbf{X}(s-1) .
$$

In formulae (18) we used the following designation for the vector $\mathbf{X}(s)$ :

$$
\mathbf{X}(s)=\left(\begin{array}{c}
X_{0}(s) \\
X_{1}(s) \\
X_{2}(s) \\
X_{3}(s)
\end{array}\right)=\left(\begin{array}{c}
\left\langle n^{\prime} \kappa^{\prime}\left|r^{s}\right| n \kappa\right\rangle \\
\left\langle n^{\prime} \kappa^{\prime}\left|\sigma_{x} r^{s}\right| n \kappa\right\rangle \\
\left\langle n^{\prime} \kappa^{\prime}\left|l \sigma_{y} r^{s}\right| n \kappa\right\rangle \\
\left\langle n^{\prime} \kappa^{\prime}\left|\sigma_{z} r^{s}\right| n \kappa\right\rangle
\end{array}\right) .
$$

Elements of the matrix $A(s)$ are respectively equal to

$$
\begin{aligned}
& a_{11}:=0, \quad a_{12}:=\frac{\left(k^{\prime}-k\right)}{\left(E_{n^{\prime} k^{\prime}}-E_{n k}\right)}, \\
& a_{13}:=-\frac{s}{\left(E_{n^{\prime} k^{\prime}}-E_{n k}\right)}, \quad a_{14}:=0, \\
& a_{21}:=\frac{\left(k^{\prime}+k\right)}{\left(E_{n^{\prime} k^{\prime}}+E_{n k}\right)}, \quad a_{22}:=-\frac{2(Z \alpha)}{\left(E_{n^{\prime} k^{\prime}}+E_{n k}\right)}, \\
& a_{23}:=0, \quad a_{24}:=-\frac{s}{\left(E_{n^{\prime} k^{\prime}}+E_{n k}\right)}, \\
& a_{31}:=\frac{2 m\left(k^{\prime}+k\right)}{\left(E_{n^{\prime} k^{\prime}}-E_{n k}\right)}+\frac{\left(E_{n^{\prime} k^{\prime}}^{2}-E_{n k}^{2}\right)}{a_{32}}:=-\frac{4 m(Z \alpha)}{\left(E_{n^{\prime} k^{\prime}}^{2}-E_{n k}^{2}\right)}, \\
& a_{33}:=0, \quad a_{34}:=-\frac{\left(k^{\prime}+k\right)}{\left(E_{n^{\prime} k^{\prime}}-E_{n k}\right)}-\frac{2 m s}{\left(E_{n^{\prime} k^{\prime}}^{2}-E_{n k}^{2}\right)}, \\
& a_{41}:=0, \quad a_{42}:=\frac{s}{\left(E_{n^{\prime} k^{\prime}}+E_{n k}\right)}+\frac{2 m\left(k^{\prime}-k\right)}{\left(E_{n^{\prime} k^{\prime}}^{2}-E_{n k}^{2}\right)}, \\
& a_{43}:=-\frac{\left(k^{\prime}-k\right)}{\left(E_{n^{\prime} k^{\prime}}+E_{n k}\right)}-\frac{2 m s}{\left(E_{n^{\prime} k^{\prime}}^{2}-E_{n k}^{2}\right)}, \\
& a_{44}:=-\frac{2(Z \alpha)}{\left(E_{n^{\prime} k^{\prime}}+E_{n k}\right)} .
\end{aligned}
$$

Note that in the matrix $A(s)$ there are five zero and two equal non-zero elements: $a_{11}=a_{14}=a_{23}=a_{33}=a_{41}=0 ; a_{22}=$ $a_{44} \neq 0$. Repeatedly using formula (18) one can obtain the following expression:

$$
\mathbf{X}(s)=\prod_{j=1}^{j=s} A(j) \cdot \mathbf{X}(0),
$$

where

$$
\mathbf{X}(0)=\left(\begin{array}{c}
0 \\
\left\langle n^{\prime} \kappa^{\prime}\left|\sigma_{x}\right| n \kappa\right\rangle \\
\left\langle n^{\prime} \kappa^{\prime}\left|l \sigma_{y}\right| n \kappa\right\rangle \\
\left\langle n^{\prime} \kappa^{\prime}\left|\sigma_{z}\right| n \kappa\right\rangle
\end{array}\right) .
$$

The upper component of the vector $\mathbf{X}(0)$ turns to zero due to the condition of orthogonality. A natural requirement which limits the applicability of the method consists of the condition of non-zero value of matrix determinant:

$$
\begin{aligned}
\Delta & =\operatorname{det} A(s) \\
& =\frac{s^{4}-2\left(k^{\prime 2}+k^{2}-2(Z \alpha)^{2}\right) s^{2}+\left(k^{\prime 2}-k^{2}\right)^{2}}{\left(E_{n^{\prime} k^{\prime}}^{2}-E_{n k}^{2}\right)^{2}} \neq 0 .
\end{aligned}
$$

Now we suppose that $s$ is a positive real, not necessarily an integer, number. It should be noted that if condition (22) is satisfied, then formula (18) is true. However in this case, repeated use of formula (18) cannot give a result in the form (20). If condition (22) is not satisfied, then we have degeneration. Then some ratios should be excluded and the relation would be realized by the matrices of smaller size. Such a situation appears, particularly, when one considers virial relations for diagonal matrix elements $[6,11,12]$. Here one has $3 \times 3$ matrices because $X_{2}(s) \equiv 0$, and in special cases they reduce to $2 \times 2$ matrices.

With virial relations (10)-(13) one connects the problem of negative powers. Let us explain the content of the problem. Let us take $\kappa$ such that integrals (6)-(9) exist. Then while $s<0$, equations (10)-(13) would not be recursive. In other words, they could not be directly used for calculating matrix elements. However if one takes into account the ratio (18), then for solving the problem of negative powers one should know the inverse matrix, that is, the existence condition (22) is satisfied:

$$
\mathbf{X}(s-1)=A^{-1}(s) \mathbf{X}(s)=B(s) \mathbf{X}(s) .
$$

Let us write out explicitly the elements of the inverse matrix $B(s)$ :

$$
\begin{aligned}
& b_{11}:=\frac{2}{\Delta}\left(k^{\prime}+k\right)\left(\left(E_{n^{\prime} k^{\prime}}-E_{n k}\right)\left(k^{\prime}-k\right)+2 m s\right), \\
& b_{12}:=\frac{1}{\Delta}\left(-2 m s^{3}-\left(k^{\prime}+k\right)\left(E_{n^{\prime} k^{\prime}}-E_{n k}\right) s^{2}+2 m\left(\left(k^{\prime}-k\right)^{2}\right.\right. \\
& \left.\left.-4(Z \alpha)^{2}\right) s+\left(k^{\prime}+k\right)\left(k^{\prime}-k\right)^{2}\left(E_{n^{\prime} k^{\prime}}+E_{n k}\right)\right), \\
& b_{13}:=\frac{1}{\Delta} s\left(E_{n^{\prime} k^{\prime}}-E_{n k}\right)\left(s^{2}-\left(k^{\prime}-k\right)^{2}+4(Z \alpha)^{2}\right) \text {, } \\
& b_{14}:=-\frac{2}{\Delta}\left(E_{n^{\prime} k^{\prime}}-E_{n k}\right)\left(k^{\prime}+k\right) s(Z \alpha) \\
& b_{21}:=\frac{1}{\Delta}\left(\left(k^{\prime}+k\right)^{2}-s^{2}\right)\left(\left(k^{\prime}-k\right)\left(E_{n^{\prime} k^{\prime}}-E_{n k}\right)+2 m s\right), \\
& b_{22}:=-\frac{2}{\Delta} s\left(\left(E_{n^{\prime} k^{\prime}}-E_{n k}\right) s+2 m\left(k^{\prime}+k\right)\right), \\
& b_{23}:=\frac{2}{\Delta}\left(k^{\prime}+k\right)\left(E_{n^{\prime} k^{\prime}}-E_{n k}\right)(Z \alpha), \\
& b_{24}:=\frac{1}{\Delta} s\left(E_{n^{\prime} k^{\prime}}+E_{n k}\right)\left(s^{2}-\left(k^{\prime}+k\right)^{2}\right), \\
& b_{31}:=-\frac{1}{\Delta}\left(\left(E_{n^{\prime} k^{\prime}}-E_{n k}\right) s^{3}+2 m\left(k^{\prime}-k\right) s^{2}-\left(\left(k^{\prime}+k\right)^{2}\right.\right. \\
& \left.-4(Z \alpha))\left(E_{n^{\prime} k^{\prime}}-E_{n k}\right) s-2 m\left(k^{\prime}-k\right)\left(\left(k^{\prime}+k\right)^{2}\right)\right), \\
& b_{32}:=\frac{2}{\Delta}\left(k^{\prime}-k\right)\left(\left(E_{n^{\prime} k^{\prime}}+E_{n k}\right) s+2 m\left(k^{\prime}+k\right)\right) \text {, } \\
& b_{33}:=\frac{2}{\Delta}\left(k^{\prime 2}-k^{2}\right)\left(E_{n^{\prime} k^{\prime}}-E_{n k}\right)(Z \alpha), \\
& b_{34}:=\frac{\left(k^{\prime}-k\right)}{\Delta}\left(E_{n^{\prime} k^{\prime}}+E_{n k}\right)\left(s^{2}-\left(k^{\prime}+k\right)^{2}\right),
\end{aligned}
$$




$$
\begin{aligned}
b_{41} & :=\frac{2}{\Delta}\left(\left(k^{\prime}-k\right)\left(E_{n^{\prime} k^{\prime}}-E_{n k}\right)+2 m s\right) s(Z \alpha), \\
b_{42} & :=-\frac{1}{\Delta}\left(s\left(E_{n^{\prime} k^{\prime}}+E_{n k}\right)+2 m\left(k^{\prime}+k\right)\right)\left(s^{2}-\left(k^{\prime}-k\right)^{2}\right), \\
b_{43} & :=\frac{\left(k^{\prime}+k\right)}{\Delta}\left(E_{n^{\prime} k^{\prime}}-E_{n k}\right)\left(s^{2}-\left(k^{\prime}-k\right)^{2}\right), \\
b_{44} & :=-\frac{2}{\Delta}\left(E_{n^{\prime} k^{\prime}}+E_{n k}\right) s^{2}(Z \alpha),
\end{aligned}
$$

Note that the matrix $B(s)$ has a more complicated structure than the matrix $A(s)$. In a general case all matrix elements are not equal to zero. However, in a particular case $k=k^{\prime}$, three elements turn to zero: $b_{32}=b_{33}=b_{34}=0$, but there are no other equal elements. Let us stress once more that formulae (18) and (23) are valid not only for an integer but also for any real values of $s$, provided that condition (22) is satisfied. This condition may be violated even by nondiagonal matrix elements, in which case we have degeneracy. In certain circumstances it is necessary to obtain some relations between the matrix elements with different powers in order to derive the asymptotic formulae. In the next section we take the calculation probabilities of forbidden M1 transitions in hydrogen atom and light hydrogen-like ions as an example and show how to do that by virtue of formula (18).

\section{Probabilities of forbidden magnetic-dipole transitions in the hydrogen atom and light hydrogen-like ions}

The magnetic-dipole transitions between the states $n j l$ and $n^{\prime} j l$ in the hydrogen atom and light hydrogen-like ions belong to the most strongly forbidden transitions in the optical range. Interest in these transitions was aroused in connection with the theoretical and experimental studies of the parity-violation effects in atoms. The magnitude of the parity-violation effect in these transitions is proportional to the ratio between the probability amplitudes for the electric-dipole transition allowed due to parity violation and the forbidden magneticdipole transition. If the latter transition is strongly forbidden, the effect becomes more pronounced [14]. The general expression for the degree of parity violation in a process occurring via an arbitrary $n \mathrm{~s}_{1 / 2}-n^{\prime} \mathrm{s}_{1 / 2}$ forbidden M1 transition is [15]

$$
\begin{gathered}
P_{n \mathrm{~s}_{1 / 2} ; n^{\prime} \mathrm{s}_{1 / 2}}=2 \cdot \frac{\left\langle n^{\prime} \mathrm{p}_{1 / 2}\left|H_{W}\right| n^{\prime} \mathrm{s}_{1 / 2}\right\rangle}{\left|E_{n^{\prime} \mathrm{p}_{1 / 2}}-E_{n^{\prime} \mathrm{s}_{1 / 2}}\right|} \sqrt{\frac{W_{n \mathrm{~s}_{1 / 2} ; n^{\prime} \mathrm{p}_{1 / 2}}^{(E 1)}}{W_{n \mathrm{~s}_{1 / 2} ; n^{\prime} \mathrm{s}_{1 / 2}}^{(M 1)}}} \\
+2 \cdot \frac{\left\langle n \mathrm{p}_{1 / 2}\left|H_{W}\right| n \mathrm{~s}_{1 / 2}\right\rangle}{\left|E_{n \mathrm{p}_{1 / 2}}-E_{n \mathrm{~s}_{1 / 2}}\right|} \sqrt{\frac{W_{n \mathrm{p}_{1 / 2} ; n^{\prime} \mathrm{s}_{1 / 2}}^{(E 1)}}{W_{n \mathrm{~s}_{1 / 2} ; n^{\prime} \mathrm{s}_{1 / 2}}^{(M 1)}}} .
\end{gathered}
$$

Here, $H_{W}$ is the effective Hamiltonian of a weak interaction and $n$ is the principal quantum number. An analogous expression for the degree of parity violation in a process occurring via an arbitrary $n \mathrm{p}_{1 / 2}-n^{\prime} \mathrm{p}_{1 / 2}$ forbidden M1 transition is obtained from expression (24) by replacing $W_{n s_{1 / 2} ; n^{\prime} \mathrm{s}_{1 / 2}}^{(M 1)}$ in the denominator under the radical sign by $W_{n \mathrm{p}_{1 / 2} ; n^{\prime} \mathrm{p}_{1 / 2}}^{(M 1)}$. It is methodically expedient to obtain general formulae at first for all the probabilities of any forbidden M1 transition, and then those which one needed.
In the nonrelativistic limit $\alpha Z \ll 1$, the probability of magnetic-dipole transitions between the $n j l$ and $n^{\prime} j^{\prime} l^{\prime}$ levels of single-electron atoms is given by expression [16]:

$$
W_{n^{\prime} j^{\prime} l^{\prime} ; n j l}^{(M 1)}=\frac{4}{3} \frac{\omega^{3}}{(2 j+1)}\left|\left\langle n^{\prime} j^{\prime} l^{\prime}|| \mu|| n j l\right\rangle\right|^{2},
$$

where $\left\langle n^{\prime} j^{\prime} l^{\prime}\|\mu\| n j l\right\rangle$ is the reduced matrix element of the magnetic dipole operator $\mu$ of the electron and $\omega$ is the transition frequency. Expression (25) is, however, inconvenient to calculate the M1 transition probabilities in our case when $n \neq n^{\prime}$. The reason is that the operator $\mu$ only depends on the spin variables, and therefore the matrix element in (25) should vanish because the radial wavefunctions $R_{n^{\prime} l^{\prime}}$ and $R_{n l}$ are orthogonal. To obtain a nonzero value, it is necessary to take into account the relativistic corrections to the wavefunctions. However, it is also necessary to consider the corrections to the operator itself. Ignoring the latter led to an error in the first calculation of the $1 s_{1 / 2}-2 s_{1 / 2}$ transition probability in the hydrogen atom carried out by Breit and Teller [17]. This error was eliminated subsequently by Drake [18].

Therefore, we will use another, completely relativistic, expression for the probability of the emission of the magnetic dipole photon [19], i.e.

$$
W_{n^{\prime} j l ; n j l}^{(M 1)}=\frac{1}{\pi(2 j+1)}\left|\left\langle n^{\prime} j l|| v^{M 1}|| n j l\right\rangle\right|^{2} .
$$

Here, $\left\langle n^{\prime} j l\left\|v^{M 1}\right\| n j l\right\rangle$ is a reduced matrix element of magnetic-dipole emission operator:

$$
\begin{aligned}
& \left\langle n^{\prime} j l\left\|v^{M 1}\right\| n j l\right\rangle \\
& \quad=\sqrt{\frac{\alpha \omega}{2 \pi}} \int_{0}^{\infty}\left[F_{n^{\prime} k}(r) G_{n k}(r)+G_{n^{\prime} k}(r) F_{n k}(r)\right] g_{1}(\omega r) \mathrm{d} r .
\end{aligned}
$$

The upper and lower components of a Dirac bispinor in the Coulomb field is represented as follows [19]:

$$
\begin{aligned}
& \left.\begin{array}{l}
G_{n k}(r) \\
F_{n k}(r)
\end{array}\right\}=\frac{ \pm(2 \lambda)^{1 / 2}}{\Gamma(2 \gamma+1)}\left[\frac{\left(m_{e} \pm E_{n k}\right) \Gamma\left(n_{r}+2 \gamma+1\right)}{\frac{4 Z \alpha m_{e}^{2}}{\lambda}\left(\frac{Z \alpha m_{e}}{\lambda}-\kappa\right) n_{r} !}\right]^{1 / 2} \\
& \times(2 \lambda r)^{\gamma} \mathrm{e}^{-\lambda r}\left\{\left(\frac{Z \alpha m_{e}}{\lambda}-\kappa\right) F\left(-n_{r}, 2 \gamma+1 ; 2 \lambda r\right)\right. \\
& \left.\mp n_{r} F\left(1-n_{r}, 2 \gamma+1 ; 2 \lambda r\right)\right\} \text {, }
\end{aligned}
$$

where the upper and lower signs refer to $G$ and $F$, respectively; $\lambda=\sqrt{m_{e}^{2}-E_{n k}^{2}}, \gamma=\sqrt{\kappa^{2}-(Z \alpha)^{2}}, n_{r}$ is the radial quantum number, $m_{e}$ is the electron mass; $Z$ is the nuclear charge; and $F$ is a confluent hypergeometric function.

In the nonrelativistic limit, i.e. $(\alpha Z) \rightarrow 0$, the argument of function $g_{1}(\omega r)$ becomes small: $\omega \sim m_{e}(\alpha Z)^{2}$, the average value of the radius vector $|\vec{r}| \sim \frac{1}{m_{e}(\alpha Z)}$; consequently $\omega r \sim(\alpha Z)$. To solve our problem, it suffices to retain two terms in the expansion of this function:

$$
g_{1}(\omega r)=\frac{4 \pi}{3} \omega r-\frac{2 \pi}{15}(\omega r)^{3}+O(\omega r)^{5} .
$$


The second term in this formula gives Drake's correction for the reduced matrix element. Substituting (29) into (27), we obtain

$$
\left\langle n^{\prime} j l\left\|v^{M 1}\right\| n j l\right\rangle=\sqrt{\frac{\alpha \omega}{2 \pi}}\left[\frac{4 \pi}{3} \omega X_{1}(1)-\frac{2 \pi}{15} \omega^{3} X_{1}(3)\right] .
$$

All integrals through which $X_{1}(1)$ and $X_{1}(3)$ are expressed are of one type (see a mathematical appendix in [20]):

$J_{\gamma}^{s, 0}\left(\alpha, \alpha^{\prime}\right)=\int_{0}^{\infty} \mathrm{e}^{-\left(\lambda+\lambda^{\prime}\right) z} z^{\gamma-1+s} F(\alpha, \gamma, 2 \lambda z) F\left(\alpha^{\prime}, \gamma, 2 \lambda^{\prime} z\right) \mathrm{d} z$ with $s=1$ and $s=3$. In principle, to calculate them, we can use the recurrence formula of Gordon [21] (see also [20]):

$$
\begin{aligned}
& J_{\gamma}^{s+1,0}\left(\alpha, \alpha^{\prime}\right)=\frac{1}{\lambda^{2}-\lambda^{\prime 2}}\left\{\left[\gamma\left(\lambda-\lambda^{\prime}\right)-2 \lambda \alpha+2 \lambda^{\prime} \alpha^{\prime}-2 \lambda^{\prime} s\right]\right. \\
& \quad \times J_{\gamma}^{s, 0}\left(\alpha, \alpha^{\prime}\right)+s\left(\gamma-1+s-2 \alpha^{\prime}\right) J_{\gamma}^{s-1,0}\left(\alpha, \alpha^{\prime}\right) \\
& \left.\quad+2 \alpha^{\prime} s J_{\gamma}^{s-1,0}\left(\alpha, \alpha^{\prime}+1\right)\right\} .
\end{aligned}
$$

This formula allows us to reduce any integral of the form $J_{\gamma}^{s, 0}\left(\alpha, \alpha^{\prime}\right)$ to the integral $J_{\gamma}^{0,0}\left(\alpha, \alpha^{\prime}\right)$, which is expressed through the Gauss hypergeometric function $F\left(\alpha, \alpha^{\prime}, \gamma,-4 \lambda \lambda^{\prime} /\left(\lambda-\lambda^{\prime}\right)^{2}\right)$. However, this method of calculation gives a cumbersome expression for the reduced matrix element (30) which will contain eight hypergeometric functions. It is difficult to simplify.

On the other hand, if we use formulas (20) and (21) for calculating $X_{1}(1)$ and $X_{1}(3)$ :

$$
X_{1}(1)=(A(1) \mathbf{X}(0))_{1}, \quad X_{1}(3)=(A(3) A(2) A(1) \mathbf{X}(0))_{1},
$$

then the reduced matrix element in (30) is represented as a linear combination of four hypergeometric functions. This expression can be simplified by using the adjacency relations [22]:

$$
\begin{gathered}
(\beta-\alpha) F(\alpha, \beta, \gamma ; z)+\alpha F(\alpha+1, \beta, \gamma ; z) \\
-\beta F(\alpha, \beta+1, \gamma ; z)=0,
\end{gathered}
$$

$$
\begin{aligned}
& (\beta-\alpha)(1-z) F(\alpha, \beta, \gamma ; z)-(\gamma-\alpha) F(\alpha-1, \beta, \gamma ; z) \\
& \quad+(\gamma-\beta) F(\alpha, \beta-1, \gamma ; z)=0
\end{aligned}
$$

If we substitute the result in (26) and pass to the limit $(\alpha Z) \rightarrow$ 0 , we obtain the general expressions for the probabilities of the forbidden M1 transition in the lowest order in $(\alpha Z)$. These expressions have different forms for different values of the total angular momentum.

1. For $j=l+1 / 2$ (referred to as spin up, which is indicated by the corresponding arrow),

$$
\begin{gathered}
W_{\uparrow}^{(M 1)}=\frac{8}{225} C_{n+l}^{2 l+1} C_{n^{\prime}+l}^{2 l+1} \frac{16^{l}(4 l+5)^{2}\left(n n^{\prime}\right)^{(2 l-6)}\left(n+n^{\prime}\right)^{2}}{(l+1)\left(n-n^{\prime}\right)^{4 l}} \\
\times\left(\frac{n-n^{\prime}}{n-n^{\prime}}\right)^{2 n+2 n^{\prime}-1} F^{2}\left(-n+l+1,-n^{\prime}+l+1,\right. \\
\left.2 l+2 ;-\frac{4 n n^{\prime}}{\left(n-n^{\prime}\right)^{2}}\right) m_{e} \alpha(\alpha Z)^{10},
\end{gathered}
$$

where $C_{n+l}^{2 l+1}$ and $C_{n^{\prime}+l}^{2 l+1}$ are the binomial coefficients.
2. For $j=l-1 / 2$ (spin down),

$$
\begin{aligned}
W_{\downarrow}^{(M 1)} & =\frac{8}{225} C_{n+l}^{2 l+1} C_{n^{\prime}+l}^{2 l+1} \frac{16^{l}(4 l-1)^{2}\left(n n^{\prime}\right)^{(2 l-6)}\left(n+n^{\prime}\right)^{2}}{l\left(n-n^{\prime}\right)^{4 l}} \\
& \times\left(\frac{n-n^{\prime}}{n-n^{\prime}}\right)^{2 n+2 n^{\prime}-1} F^{2}\left(-n+l+1,-n^{\prime}+l+1,\right. \\
2 l & \left.+2 ;-\frac{4 n n^{\prime}}{\left(n-n^{\prime}\right)^{2}}\right) m_{e} \alpha(\alpha Z)^{10} .
\end{aligned}
$$

For $l=0$, the following expression for the probability of M1 transitions between all possible $n \mathrm{~s}_{1 / 2}$ levels can be obtained from formula (34):

$$
\begin{aligned}
& W_{n \mathrm{~s} 1 / 2 ; n^{\prime} \mathrm{s} 1 / 2}^{(M 1)}=\frac{8}{9} \frac{\left(n+n^{\prime}\right)^{2}}{n^{5} n^{\prime}}\left(\frac{n-n^{\prime}}{n-n^{\prime}}\right)^{2 n+2 n^{\prime}-1} \\
& \quad \times F^{2}\left(1-n, 1-n^{\prime}, 2 ;-\frac{4 n n^{\prime}}{\left(n-n^{\prime}\right)^{2}}\right) m_{e} \alpha(\alpha Z)^{10} .
\end{aligned}
$$

For $n^{\prime}=2$, expression (36) gives the known Drake formula [18]

$$
W_{1 \mathrm{~s}_{1 / 2} ; 2 \mathrm{~s}_{1 / 2}}^{(M 1)}=\frac{1}{972} m_{e} \alpha(\alpha Z)^{10}
$$

It should be noted that expression (36) can also be obtained by using the effective potential method, described in [14] and [23]. All the formulas necessary to investigate the parity-violation effects in hydrogen and light $\mathrm{H}$-like ions can be derived from (34) and (35) by substitution of respective quantum numbers. Such a list was first presented in [24] by complicated calculations involving Gordon's formula.

\section{Conclusion}

In this paper we have shown that the set of non-diagonal elements $\mathbf{X}(s)$ is related to the set $\mathbf{X}(s-1)$ by $4 \times 4$ matrices according to formulae (18) and (23). Condition (22) of applicability was also established for this representation. On one hand, formulae (18) and (23) may be useful for the derivation of diverse asymptotic relations when one needs to connect matrix elements with different powers. We have illustrated our method with the calculation of the probability of forbidden M1 transitions. On the other hand, in the calculations of higher-order corrections to various physical quantities, one needs to evaluate the sums

$$
\left|i, s, \kappa^{\prime}, n \kappa\right\rangle=\sum_{n^{\prime}}^{\left(E_{n^{\prime} \kappa^{\prime}} \neq E_{n \kappa}\right)} \frac{\left|n^{\prime} \kappa^{\prime}\right\rangle\left\langle n^{\prime} \kappa^{\prime}\left|R_{i}^{s}\right| n k\right\rangle}{E_{n \kappa}-E_{n^{\prime} \kappa^{\prime}}},
$$

where $R_{0}^{s}=r^{s}, R_{1}^{s}=\sigma_{x} r^{s}, R_{2}^{s}=\imath \sigma_{y} r^{s}, R_{3}^{s}=\sigma_{z} r^{s}$. In papers $[5,6]$ the virial relations were employed to evaluate sums (37) in some important special cases. In principle, it would be interesting to have simple and compact expressions for all such sums with arbitrary powers $s$. Hopefully, the matrix representations (18) and (23) might be useful for this. It would also be very interesting to make an attempt to write the non-diagonal matrix elements as sums of special generalized hypergeometric functions ${ }_{q} F_{p}$ with the integers $p$ and $q$ as in the works of Suslov $[11,12]$. It is important for a better 
understanding of the structure of relativistic Coulomb integrals.

The author believes that the mathematical results obtained in this paper are not only natural and elegant but also will be useful in the current theory of hydrogen-like heavy ions and other exotic relativistic Coulomb systems.

\section{Acknowledgments}

The author is grateful to Leonti N Labzowsky, Vladimir M Shabaev, Vladimir A Yerokhin and Alexey V Golovnev for important comments and encouragement. The referees' suggestions are also very much appreciated.

\section{References}

[1] Epstein J N and Eppstein S T 1962 Some applications of hypervirial theorems to the calculation of average values Am. J. Phys. 30226

[2] Epstein S T, Epstein J N and Kennedy B 1967 Recursion relations for Coulomb matrix elements J. Math. Phys. 81747

[3] Blanchard P 1974 A new recurrence relation for hydrogenic radial matrix elements J. Phys. B: At. Mol. Phys. 7993

[4] Shabaev V M 1984 Recurrence formulas and some exact relations for radial integrals with Dirac and Schrödinger wave functions Vestnik Leningradskogo Universiteta, Seria 4: Fizika, Khimiya 1 15-9 (in Russian)

[5] Shabaev V M 1991 Generalizations of the virial relations for the Dirac equation in a central field and their applications to the Coulomb field J. Phys. B: At. Mol. Opt. Phys. 244479

[6] Shabaev V M 2003 Virial relations for the Dirac equation and their applications to calculations of hydrogen-like atoms Precision Physics of Simple Atomic Systems (Lecture Notes in Physics vol 627) (Berlin: Springer) p 97 arXiv:physics/0211087

[7] Adkins G S 2008 Dirac-Coulomb energy levels and expectation values Am. J. Phys. 76579

[8] Yerokhin V A, Indelicato P and Shabaev V M 2004 Evaluation of the self-energy correction to the $g$ factor of $S$ states in H-like ions Phys Rev. A 69052503
[9] Ivanov V G, Karshenboim S G and Lee R N 2009 Electron shielding of the nuclear magnetic moment in a hydrogenlike atom Phys. Rev. A 79012512

[10] Yerokhin V A and Jentschura U D 2010 Self-energy correction to the hyperfine splitting and the electron $g$ factor in hydrogenlike ions Phys. Rev. A 81012502

[11] Suslov S K 2009 Expectation values in relativistic Coulomb problems J. Phys. B: At. Mol. Opt. Phys. 42185003

[12] Suslov S K 2010 Relativistic Kramers-Pasternac recurrence relations J. Phys. B: At. Mol. Opt. Phys. 43074006

[13] Drake G W F and Goldman S P 1981 Application of discrete-basic-set methods to the Dirac equation Phys. Rev. A 232093

[14] Khriplovich I B 1988 Parity Nonconservation in Atomic Phenomena (Moscow: Nauka) (in Russian)

[15] Puchkov A M and Labzowsky L N 2010 Parity violation effects in hydrogen atom in forbidden magnetic-dipole transitions Opt. Spektrosk. 108713

[16] Berestetskii V B, Lifshiz E M and Pitaevskii L P 1989 Course of Theoretical Physics: vol 4. Quantum Electrodynamics (Moscow: Nauka)

Berestetskii V B, Lifshiz E M and Pitaevskii L P 1982 Course of Theoretical Physics: vol 4. Quantum Electrodynamics (Oxford: Pergamon)

[17] Breit G and Teller E 1940 Metastability of hydrogen and helium levels Astrophys. J. 91215

[18] Drake G W F 1971 Theory of relativistic magnetic dipole transitions: lifetime of metastable $2^{3} \mathrm{~S}$ state of the heliumlike ions Phys. Rev. A 3908

[19] Labzowsky L N 1996 The Theory of the Atom: Quantum Electrodynamics of Electron Shells and Radiation Processes (Moscow: Nauka) (in Russian)

[20] Landau L D and Lifshitz E M 1977 Quantum Mechanics: Non-Relativistic Theory 3rd edn (Oxford: Pergamon)

[21] Gordon W 1929 Zur Berechnung der Matrizen beim Wasserstoffatom Ann. Phys., Lpz. 21031

[22] Erdelyi A 1953 Higher Transcendental Functions vols I-III, ed A Erdelyi (New York: McGraw-Hill)

[23] Sucher J 1978 Magnetic dipole transitions in atomic and particle physics: ions and psions Rep. Prog. Phys. 411783

[24] Puchkov A M and Labzowsky L N 2009 Probabilities of forbidden magnetic-dipole transitions in the hydrogen atom and hydrogenlike ions Opt. Spektrosk. 106181 